\title{
ETHICS IN ETHICS COMMITTEES
}

\section{Blanket informed consent for retrospective studies is justified}

\author{
PRABHA DESIKAN
}

Department of Microbiology, Bhopal Memorial Hospital and Research Centre, Raisen Bypass road, Karond, Bhopal ,Madhya Pradesh 462038 INDIA

email:prabhadesikan@yahoo.com Email - prabhadesikan@yahoo.com

As with most issues associated with ethics in medical research, this question (1) has the potential to evoke passionate responses, both justifying and disparaging the concept. My thoughts on the issue have to do with my situation in various capacities in a healthcare set up.

\section{As a laboratory person}

Many laboratory diagnostic tests have their own limitations. Even after ruling out technical and inter-observer related issues for a test result, it may be difficult to explain the clinical significance of a positive diagnostic test in the absence of classical signs and symptoms of the disease under question, due to lack of published studies addressing the issue. Similar questions arise with a negative test result, when the pre-test possibility of a positive test result is extremely high. It is difficult to explain to a patient (and clinicians) that we do not have cut and dried answers to diagnostic dilemmas. Many times, it is only possible to hypothesise about factors in the individual patient's internal milieu that may interfere with the test conditions. We can attempt to answer these dilemmas by carrying out a large population-based study with defined inclusion and exclusion criteria. A prospective study would be ideal, but follow-up may run into years, and sometimes decades. Such timeframes may not be practical in terms of sustaining clinical or research interest for such a long period, or for obtaining grants from tight-fisted funding agencies. It certainly is not useful for the patient in question, who has to be given appropriate therapy for an undiagnosed illness. A retrospective study might solve the issue - if samples are stored properly. The results of such a study will have a direct influence on contemporary clinical practice. It may be able to provide answers in a timeframe that is relevant to the patient's needs. Since it may not be possible to have an idea about the aims of a potential retrospective study at the time a patient visits a healthcare facility, it may make logistical and economic sense to take blanket consent from the patient at the time of the first visit.

\section{As a potential (and past) patient}

I certainly would not have any issues with my clinical profile and samples being used for a retrospective study, provided that my confidentiality is maintained. My perception is that I have no right to deny use of my clinical material when it is being used for the larger interests of the community. Wilful restriction of advancement of medical knowledge - for reasons of potential personal interest - is selfish, at best. Though I quite agree that this is an individual perception, I wonder whether the large numbers of patients attending the clinics of public hospitals would even give the issue of blanket consent a second thought. As a patient, my main concern would be (and has been) to get better. If my treating physician would like my consent to use the knowledge gained from my illness for a retrospective study, my instinctive reaction would be to tell him / her to get it over with, and to continue to help me recover from my illness. If $\mathrm{I}$, as an educated healthcare professional who is aware of my rights, feel this way, it is a safe bet that most people - across all socioeconomic strata - would do so too.

\section{As the secretary of the IRB of my institute}

While retrospective studies have their uses, many of them have issues with ill-defined inclusion and exclusion criteria. This is because it is difficult to find well-defined cohorts to fit stringent inclusion and exclusion criteria in a retrospective study. In addition, I find that vested interests need to be guarded against in a retrospective study. Therefore, more than consent from individual patient, what needs to come under the scanner is the vigilance of the Institutional Review Board (IRB). If the aims and objectives of retrospective studies are above board, then taking blanket consent for retrospective studies should not be an issue.

\section{As a researcher}

Planning a project requires a tremendous amount of input from the principal investigator. Starting from writing to various funding agencies, to finding committed collaborators, it is an uphill task all the way. Sometimes, overzealous objections from some of the members of an IRB come across as (hopefully unintended) attempts to hinder research. As a researcher, I could do with fewer obstacles to overcome for me to carry out meaningful, clinically useful research. Obtaining blanket consent for retrospective studies would give me a breather.

To sum up, I feel that there is a scientific need to broaden the consent obtained. Ethicists must recognise that research subjects, when well informed, have a right to participate even in broadly-defined research. Having said that, it would be reasonable to give the individual the option to refuse consent at a later date, if he or she feels morally obliged to do so.

\section{Reference}

1. Jesani A. About student research and blanket consent from patients. Indian J Med Ethics. 2009 Oct-Dec. 6(4): 216-8. 